\title{
Predictors and risk factors for intestinal necrosis in patients with mesenteric ischemia
}

\author{
Hongwei Zhao", Yiting Meng", Peng Zhang, Qian Zhang, Feng Wang, Yuanxin Li \\ Department of Gastrointestinal Surgery, Beijing Tsinghua Changgung Hospital, School of Clinical Medicine, Tsinghua University, Beijing, China \\ Contributions: (I) Conception and design: H Zhao; (II) Administrative support: Y Li; (III) Provision of study materials or patients: P Zhang; (IV) \\ Collection and assembly of data: Y Meng; (V) Data analysis and interpretation: Q Zhang; (VI) Manuscript writing: All authors; (VII) Final approval \\ of manuscript: All authors. \\ "These authors contributed equally to this work. \\ Correspondence to: Yuanxin Li, MD. Department of Gastrointestinal Surgery, Beijing Tsinghua Changgung Hospital, School of Clinical Medicine, \\ Tsinghua University, No.168 Litang Road, Changping District, Beijing 102218, China. Email: lyxa01633@btch.edu.cn.
}

Background: Mesenteric ischemia results in blood flow that is insufficient to meet metabolic demands and subsequent dysfunction of visceral organs, including arterial obstruction and venous thrombosis. Sustained mesenteric ischemia exhausts the ability of capillaries to provide oxygen and initiate an inflammatory reaction, and eventually leads to intestinal mucosal necrosis, a serious and potentially life-threatening condition. Therefore, it is essential that the predictors and risk factors for intestinal necrosis in patients with mesenteric thrombus are explored.

Methods: This study retrospectively enrolled 41 patients with mesenteric ischemia (including mesenteric vein embolism, mesenteric artery thrombosis, and portal vein thrombosis) who were admitted to the Department of Gastrointestinal Surgery, Beijing Tsinghua Changgung Hospital between May 2016 and October 2019; of the patients, 18 were further diagnosed with intestinal necrosis. Comparisons of symptoms, computed tomography angiography (CTA) features, and laboratory examination results were performed between mesenteric ischemia patients with and without intestinal necrosis.

Results: White blood cell count showed an excellent predictive ability for intestinal necrosis in patients with mesenteric ischemia, with an area under the receiver operating characteristic (ROC) curve of 0.772 $(\mathrm{P}=0.009)$. The four CTA features [pneumatosis $(\mathrm{P}=0.016)$, intestinal swelling $(\mathrm{P}=0.006)$, ascitic fluid $(\mathrm{P}<0.001)$, and decreased intestinal wall enhancement $(\mathrm{P}=0.004)]$ differed significantly between patients with and without intestinal necrosis. Peritonitis showed a strong association with intestinal necrosis $(\mathrm{P}=0.006)$ in the univariate analysis, and multivariate analysis further showed their association [odds ratio (OR): 8.53; 95\% CI: 1.46-49.81; P=0.017].

Conclusions: White blood cell count is a potential predictor of intestinal necrosis. Peritonitis is a possible risk factor for intestinal necrosis in patients with mesenteric ischemia. A multi-center prospective study with a larger sample size needs to be performed to further investigate these findings.

Keywords: Mesenteric ischemia; intestinal necrosis; risk factors; white blood cell; peritonitis

Submitted Dec 01, 2020. Accepted for publication Jan 26, 2021.

doi: 10.21037/atm-20-8154

View this article at: http://dx.doi.org/10.21037/atm-20-8154

\section{Introduction}

Mesenteric ischemia results in blood flow that is insufficient to meet metabolic demands and subsequent dysfunction of visceral organs, including arterial obstruction and venous thrombosis (1-3). Arterial obstruction, which has both acute and chronic forms, is considered to be the most common cause of mesenteric ischemia (1). Acute mesenteric ischemia (AMI), with 40-50\% cases resulting from embolic occlusion 
and $20-35 \%$ cases resulting from thrombotic occlusion of a previously stenotic mesenteric vessel, has a high overall mortality rate, which is in excess of $80 \%(1,2,4)$. The majority of cases of chronic mesenteric ischemia (>90\%) are related to progressive atherosclerotic disease (1).

Mesenteric venous thrombosis (MVT) impairs venous outflow and causes visceral edema (1,5). Among patients with mesenteric ischemia, the incidence rate of MVT is $5-15 \%$. MVT mostly occurs in the superior mesenteric vein and rarely in the inferior mesenteric vein $(6,7)$. Thrombosis can occur in large mesenteric veins due to several reasons, including idiopathic thrombosis, thrombophilia, trauma, and local inflammatory changes (6-8). A hypercoagulable state is usually a result of surgery or an inflammatory disease, and significantly promotes the formation of thrombi in small vessels $(6,9)$.

In a resting state, the bowl can tolerate a marked reduction in blood flow, as only one-fifth of capillaries are needed to provide adequate oxygen delivery to tissues (9). However, during stress, oxygen extraction can be augmented by the intestinal mucosa. Sustained mesenteric ischemia exhausts the ability of capillaries to provide oxygen and initiate an inflammatory reaction, which eventually leads to intestinal mucosal necrosis, a potentially life-threatening condition. Therefore, it is essential that the predictors and risk factors for intestinal necrosis in patients with MVT are explored.

In this study, symptoms, computed tomography angiography (CTA) imaging features, and laboratory examination results were compared between mesenteric thrombus patients with and without intestinal necrosis.

We present the following article in accordance with the STROBE reporting checklist (available at http://dx.doi. org/10.21037/atm-20-8154). ${ }^{1}$

\section{Methods}

\section{Patients}

This study retrospectively reviewed 41 patients with mesenteric ischemia who were admitted to the Department of Gastrointestinal Surgery, Beijing Tsinghua Changgung Hospital between May 2016 and October 2019. Of the 41 patients enrolled, 18 were further diagnosed with intestinal necrosis.

Patients aged over 18 years old were included. The inclusion criteria were as follows: patients with mesenteric vein embolism, mesenteric artery thrombosis, or portal vein thrombosis.
Patients with acute abdominal diseases, intestinal obstruction caused by other diseases, or incomplete medical records were excluded, as were patients who refused to cooperate.

\section{Data collection}

All procedures performed in this study involving human participants were in accordance with the Declaration of Helsinki (as revised in 2013). Approval for this study was granted by the Ethics Committee of Beijing Tsinghua Changgung Hospital (No. 19033-0-01) and was performed under the protocol of this committee. Informed consent was obtained from each patient prior to their involvement in the study.

Patient clinical data including age, time elapsed from symptom onset to diagnosis, disease history, symptoms, complications, body temperature, and heart rate were recorded. Regular blood samples were collected from the patients for laboratory examination.

\section{Diagnosis}

Diagnosis of mesenteric ischemia was made on the basis of disease history, clinical symptoms, physical examination, and imaging. The diagnostic signs included intestinal injury and vascular insufficiency of the superior mesenteric vein, superior mesenteric artery, or portal vein. The diagnostic signs, including intestinal edema, seroperitoneum and vascular insufficiency of the superior mesenteric vein, superior mesenteric artery, and portal vein, were diagnosed by CTA.

\section{Assessments}

The symptoms related to abdominal pain, eating disorders, abdominal distention, nausea, vomit, hematemesis, hematochezia, diarrhea, and peritonitis were recorded.

Laboratory examinations were carried out, including for white blood cell (WBC) count, neutrophil count, $\mathrm{D}-\mathrm{D}$ dimer, lactic acid, pondus hydrogenii $(\mathrm{PH})$, creatine phosphokinase (CK), and myohemoglobin (MYO).

\section{Treatment and confirmation of diagnosis}

For patients without a sign of intestinal necrosis based on CTA diagnosis, femoral arteriography was performed to remove the intestinal thrombus (Figure 1). For patients with signs of intestinal necrosis, exploratory laparotomy 

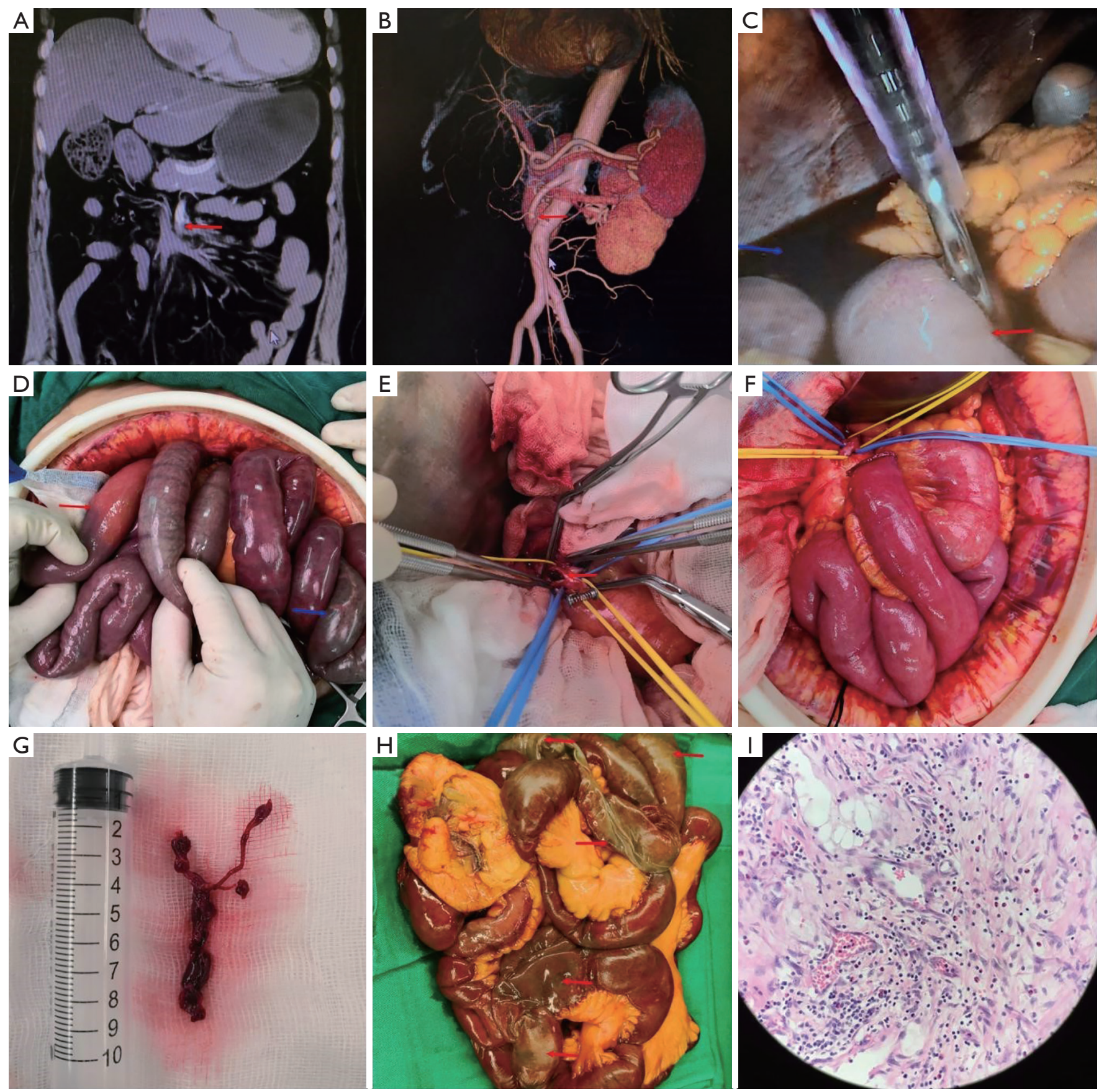

Figure 1 Diagnosis and treatment process. (A) Shows a contrast-enhanced computed tomography (CT) image of a patient's abdomen, showing superior mesenteric artery thrombosis; (B) shows revascularization with disruption of the superior mesenteric artery; (C) shows laparoscopic exploration of the patient, with intestinal necrosis and hemorrhagic effusion visible in the abdominal cavity; (D) is the necrotic small intestine, visible on laparotomy; (E) shows the process of incising the superior mesenteric artery to remove the hemorrhagic thrombus; (F) is the intestinal tract, where the blood supply recovers after thrombectomy; $(\mathrm{G})$ is a thrombus removed intraoperatively; $(\mathrm{H})$ is the resected necrotic intestine; (I) shows morphological characteristics of resected tissue $(100 \times)$. After fixed by paraformaldehyde and embedded with paraffin, the resected tissues were sliced and stained with hematoxylin and eosin (HE). 
Table 1 Basic characteristics of the patients

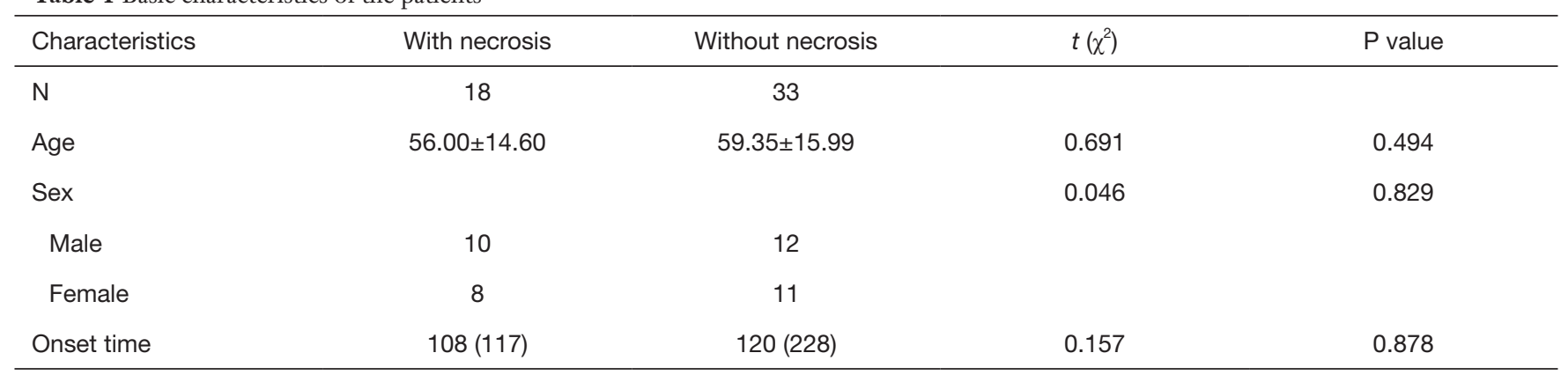

Table 2 Association of symptoms with intestinal necrosis

\begin{tabular}{lcccc}
\hline Symptoms & With necrosis & Without necrosis & $\chi^{2}$ & P value \\
\hline Eating disorders & $3(16.7 \%)$ & $9(27.3 \%)$ & 0.285 & 0.670 \\
Abdominal distention & $13(72.2 \%)$ & $17(51.5 \%)$ & 2.036 & 0.217 \\
Nausea & $11(61.1 \%)$ & $11(33.3 \%)$ & 4.413 & 1.508 \\
Vomit & $9(50.0 \%)$ & $10(30.3 \%)$ & 0.028 & 0.313 \\
Hematemesis & $2(11.1 \%)$ & $1(3.0 \%)$ & 0.965 & 1.000 \\
Hematochezia & $3(16.7 \%)$ & $8(24.2 \%)$ & 0.028 & 0.470 \\
Diarrhea & $1(5.6 \%)$ & $2(6.1 \%)$ & 8.276 & 1.000 \\
Peritonitis & $11(61.1 \%)$ & $8(24.2 \%)$ & 0.006 \\
\hline
\end{tabular}

and surgery were performed. If intestinal necrosis was confirmed in the exploratory laparotomy, resection of the necrotic intestine was carried out.

\section{Statistical analysis}

In this study, continuous variables were expressed as mean \pm standard deviation (SD) or median (interquartile range). Comparisons of means and rates between the 2 groups were performed using the $t$-test and Chi-square test, respectively. The risk factor analysis was performed using a binary logistic model. Univariate and multivariate analyses were conducted. Receiver operating characteristic (ROC) curve analysis was used to analyze the performance of factors in predicting the risk of intestinal necrosis. $\mathrm{P}<0.05$ indicated a statistically significant difference.

\section{Results}

\section{Basic characteristics of patients}

The basic characteristics of mesenteric ischemia patients with and without intestinal necrosis were compared (Table 1). No significant differences were found between the 2 groups in terms of age $(56.00 \pm 14.60$ vs. $59.35 \pm 15.99, \mathrm{P}=0.494)$, sex $(\mathrm{P}=0.829)$, or time elapsed from symptom onset to diagnosis (108 vs. 120, $\mathrm{P}=0.878$ ).

\section{Underlying risk factors for intestinal necrosis}

The associations of symptoms with intestinal necrosis were evaluated. As shown in Table 2, no significant associations were found between intestinal necrosis and eating disorders $(\mathrm{P}=0.670)$, abdominal distention $(\mathrm{P}=0.217)$, nausea ( $\mathrm{P}=0.090)$, vomiting $(\mathrm{P}=0.313)$, hematemesis $(\mathrm{P}=1.000)$, hematochezia $(\mathrm{P}=0.470)$, or diarrhea $(\mathrm{P}=1.000)$. Only peritonitis showed a strong association with intestinal necrosis $(\mathrm{P}=0.006)$.

\section{CTA features at diagnosis}

The diagnostic ability of CTA was further investigated. Four CTA diagnostic features (intestinal pneumatosis, 
Table 3 Prognostic ability of CTA for intestinal necrosis in patients with mesenteric ischemia

\begin{tabular}{|c|c|c|c|c|}
\hline Diagnostic features & With necrosis & Without necrosis & $\chi^{2}$ & $P$ value \\
\hline Intestinal swelling & $13(72.2 \%)$ & $12(36.4 \%)$ & 7.577 & 0.006 \\
\hline Ascitic fluid & $12(66.7 \%)$ & $4(12.1 \%)$ & 17.298 & $<0.001$ \\
\hline $\begin{array}{l}\text { Decreased intestinal wall } \\
\text { enhancement }\end{array}$ & $8(44.4 \%)$ & $3(9.1 \%)$ & 8.192 & 0.004 \\
\hline
\end{tabular}

CTA, computed tomography angiography.

Table 4 Predictive ability of factors for intestinal necrosis

\begin{tabular}{llll}
\hline \multirow{2}{*}{ Predictors } & \multicolumn{3}{c}{ ROC curves } \\
\cline { 2 - 4 } Time from onset to diagnosis & AUC & 95\% Cl & P value \\
Body temperature & 0.516 & $0.323-0.710$ & 0.876 \\
WBC count & 0.666 & $0.486-0.846$ & 0.122 \\
Neutrophil count & 0.772 & $0.611-0.932$ & 0.224 \\
D-dimer & 0.627 & $0.441-0.813$ & 0.540 \\
Lactic acid & 0.564 & $0.340-0.789$ & 0.467 \\
PH & 0.415 & $0.190-0.460$ & 0.572 \\
CK & 0.565 & $0.340-0.791$ & 0.375 \\
MYO & 0.607 & $0.372-0.841$ & 0.332 \\
\hline
\end{tabular}

ROC, receiver operating characteristic; AUC, area under the curve; $\mathrm{Cl}$, confidence interval; WBC, white blood cell; $\mathrm{PH}$, pondus hydrogenii; CK, creatine phosphokinase; MYO, myohemoglobin.

intestinal swelling, ascitic fluid, and decreased intestinal wall enhancement) were analyzed (Table 3). Intestinal pneumatosis was observed in $3(16.7 \%)$ patients with intestinal necrosis and $12(36.4 \%)$ patients without intestinal necrosis $(\mathrm{P}=0.016)$. Intestinal swelling was found in 13 (72.2\%) patients with intestinal necrosis and 12 (36.4\%) patients without intestinal necrosis $(\mathrm{P}=0.006)$. Ascitic fluid was observed in $12(66.7 \%)$ patients with intestinal necrosis and $4(12.1 \%)$ patients without intestinal necrosis $(\mathrm{P}<0.001)$. Decreased intestinal wall enhancement was observed in $8(44.4 \%)$ patients with intestinal necrosis and $3(9.1 \%)$ patients without intestinal necrosis $(\mathrm{P}=0.004)$. Therefore, the 4 CTA features showed significant differences between patients with and without intestinal necrosis.

\section{Underlying predictors for intestinal necrosis}

Furthermore, we analyzed the predictive ability of laboratory examination-related factors (Table 4). As shown in Figure 2, with an AUC of 0.772 , only WBC count displayed an excellent predictive ability for intestinal necrosis in patients with mesenteric ischemia $(\mathrm{P}=0.009)$. Time elapsed from symptom onset to diagnosis $(\mathrm{P}=0.876)$, body temperature $(\mathrm{P}=0.122)$, neutrophil count $(\mathrm{P}=0.224)$, $\mathrm{D}-\mathrm{D}$ dimer $(\mathrm{P}=0.540)$, lactic acid $(\mathrm{P}=0.467), \mathrm{PH}(\mathrm{P}=0.572)$, CK $(\mathrm{P}=0.375)$, and MYO $(\mathrm{P}=0.332)$ were not found to be predictive of intestinal necrosis.

\section{Multivariate analysis of risk factors for intestinal necrosis}

After the univariate analysis, a multivariate analysis of risk factors for intestinal necrosis was carried out (Table 5). Among all the factors available for multivariate analysis, only peritonitis showed a significant difference $(\mathrm{P}=0.017)$, with an odds ratio $(\mathrm{OR})$ of 8.53 . This result indicated that peritonitis is possibly a risk factor for intestinal necrosis in 

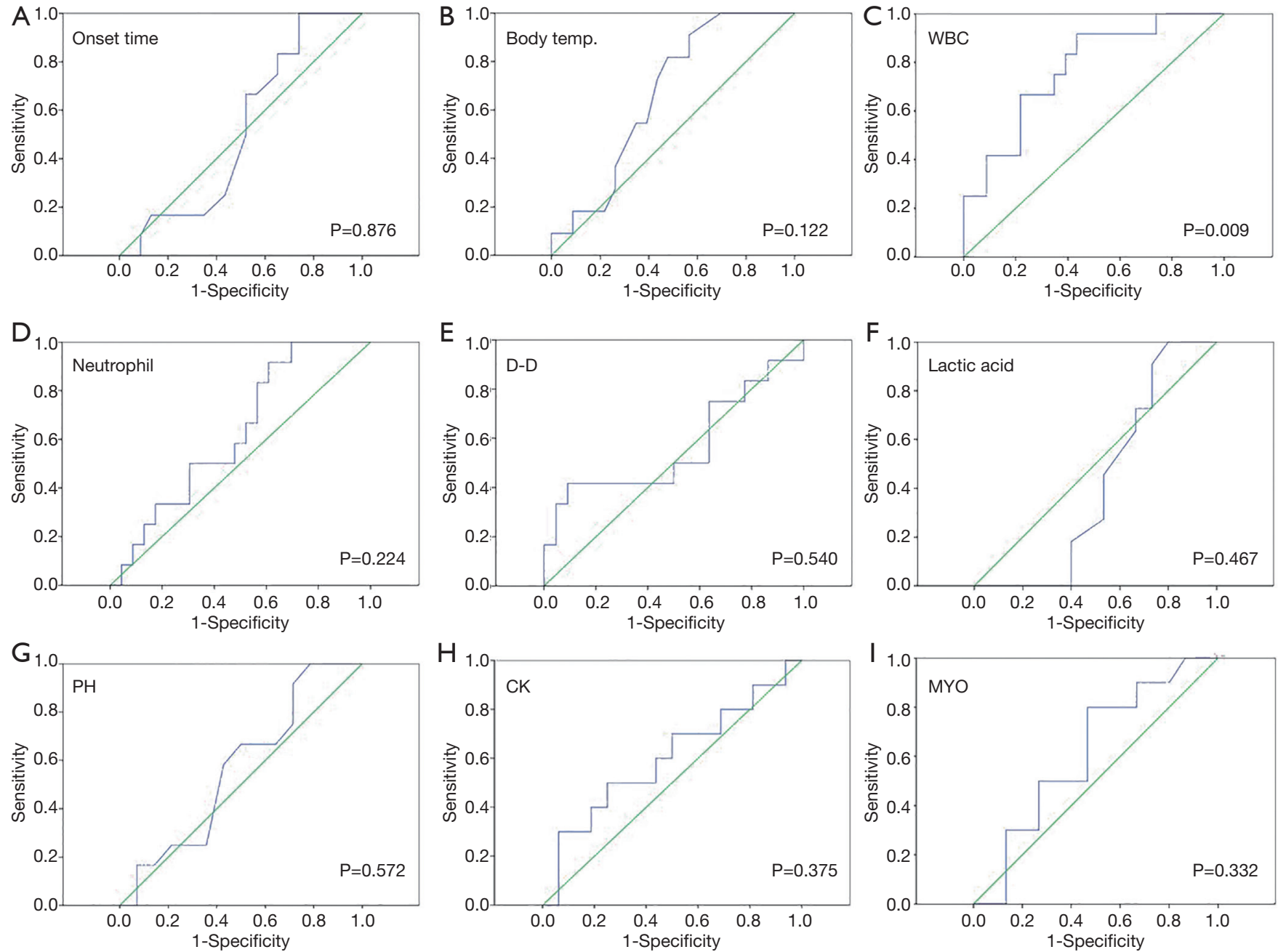

Figure 2 Receiver operating characteristic curves of indicators for intestinal necrosis. (A) Shows receiver operating characteristic curves of onset time $(\mathrm{P}=0.876)$; $(\mathrm{B})$ shows receiver operating characteristic curves of body temp $(\mathrm{P}=0.122)$; (C) shows receiver operating characteristic curves of WBC $(\mathrm{P}=0.009)$; (D) shows receiver operating characteristic curves of neutrophil ( $\mathrm{P}=0.224)$; (E) shows receiver operating characteristic curves of $\mathrm{D}-\mathrm{D}(\mathrm{P}=0.540)$; $(\mathrm{F})$ shows receiver operating characteristic curves of lactic acid $(\mathrm{P}=0.467)$; $(\mathrm{G})$ shows receiver operating characteristic curves of $\mathrm{PH}(\mathrm{P}=0.572)$; $(\mathrm{H})$ shows receiver operating characteristic curves of $\mathrm{CK}(\mathrm{P}=0.375)$. (I) shows receiver operating characteristic curves of MYO $(\mathrm{P}=0.332)$. WBC, white blood cell; D-D, D-dimer; $\mathrm{PH}$, pondus hydrogenii; $\mathrm{CK}$, creatine phosphokinase; MYO, myohemoglobin.

Table 5 Multivariate analysis of risk factors for intestinal necrosis

\begin{tabular}{|c|c|c|c|c|c|}
\hline Suspected risk factors & $\chi^{2}$ & OR & \multicolumn{2}{|c|}{$95 \% \mathrm{Cl}$} & $P$ value \\
\hline Eating disorders & 0.328 & 1.89 & 0.22 & 16.53 & 0.567 \\
\hline Vomit & 0.108 & 1.78 & 0.30 & 10.36 & 0.523 \\
\hline Hematochezia & 0.062 & 0.80 & 0.13 & 4.74 & 0.803 \\
\hline
\end{tabular}

OR, odds ratio; $\mathrm{Cl}$, confidence interval. 
patients with mesenteric ischemia.

\section{Discussion}

Mesenteric ischemia, especially AMI, is mainly caused by thromboembolic occlusive mesenteric ischemia (accounting for approximately two-thirds of cases), non-occlusive mesenteric ischemia (approximately one-sixth of cases), and MVT (approximately one-sixth of cases) (2). Patients with AMI show rapid progression, making timely diagnosis critical.

Ultrasonography (10), CT $(11,12)$, magnetic resonance angiography (11), endoscopy $(13,14)$, and catheter angiography (15) can be used for the diagnosis of mesenteric ischemia. In the past 2 decades, the development of multi-slice contrast-enhanced CT has seen it become the most important diagnostic examination method as an evolutionary transformation in the diagnosis of AMI (2). CTA has many advantages; it has high accuracy (95-100\%) and can be used to rapidly obtain vessel images with the origins and whole length, reveal the extent of stenosis or occlusion, and assess options for revascularization (1). Known specific CT features of mesenteric vein thrombosis include bowel wall thickening, intramural or mesenteric fat edema, intramural hemorrhage, the presence of air in the bowel wall or mesenteric vessels, and thrombosis with or without portal vein thrombosis (16). Meanwhile, specific CT features of mesenteric artery thrombosis include abnormal bowel wall thickness, diminished bowel wall enhancement on contrast-enhanced CT, high signal intensity with reperfusion, hypotonic bowel dilatation, and arterial defects (17). In our study, 4 specific CTA features were compared between mesenteric ischemia patients with and without intestinal necrosis. All of the cases indicated a fairly good diagnostic ability for intestinal necrosis. In patients with intestinal necrosis, intestinal pneumatosis was decreased, intestinal swelling was increased, intestinal wall enhancement was decreased, and ascitic fluid could be observed.

An increase in WBC count is associated with an inflammatory reaction caused by local disease that has spread to the entire immune system. Glenister et al. mentioned in their review that a high lactate level and white cell count in a patient with abdominal pain should raise suspicion of bowel ischemia, although they are not diagnostic (18). Moreover, Kim et al. found that levels of leukocytes were associated with bowel infarction in patients with MVT (19), and Nuzzo et al. reported an association of WBC count with intestinal necrosis in patients with AMI (20). Our results also suggested that WBC count is associated with intestinal necrosis, indicating a promising potential of $\mathrm{WBC}$ count in predicting intestinal necrosis in patients with mesenteric ischemia. Interestingly, we did not observe a significant increase in lactate level in our study, which is inconsistent with other reports $(18,20,21)$. This is probably attributable to the heterogeneity of patients and the limited number of patients admitted to a single center.

Peritonitis-the signs of which include abdominal tenderness, rebound tenderness, and rigidity-is local inflammation confined to the abdominal cavity. Corcos et al. assessed a multidisciplinary and multimodal management approach for AMI patients to improve survival and prevent intestinal failure, in which early AMI was defined as the absence of organ failures, elevated levels of blood lactate, and pneumoperitoneum/peritonitis on CT diagnosis and late AMI was considered as the presence of peritonitis and 2 other signs (22). It was indicated that peritonitis might predict a poor prognosis for AMI patients. In patients with acute superior MVT, Kim et al. found that abdominal distention and rebound tenderness were associated with bowel infarction (19). Moreover, Wang et al., who studied predictive risk factors for intestinal necrosis in patients with MVT, reported that peritonitis was associated with intestinal necrosis (23). Consistent with these findings, our results also suggest that peritonitis may be a risk factor for intestinal necrosis.

Besides early diagnosis, early preventive treatment for intestinal necrosis is also necessary. Given that the development of severe metabolic acidosis may result from intestinal necrosis, fluid resuscitation and serial monitoring of electrolytes should be used as early as possible $(24,25)$. With regard to therapy, endovascular repair and open repair are treatment options for AMI, and for chronic mesenteric ischemia, revascularization is indicated. In most cases of venous mesenteric ischemia, anticoagulation is the only therapy necessary, and heparin should be given to patients for systemic anticoagulation $(7,26)$. When severe acute MVT refractory to anticoagulation occurs, catheter-assisted thrombectomy and catheter-directed fibrinolysis can be used (27).

The main limitation of this study lies in its retrospective nature and the small number of patients involved. In future, a prospective, multi-center study would significantly increase the reliability of our conclusions. The study from our center has taken a small step toward finding predictors and risk factors for intestinal necrosis in patients with 
mesenteric ischemia.

\section{Conclusions}

WBC count is associated with and is potentially a predictor of intestinal necrosis. Peritonitis is a possible risk factor for intestinal necrosis in patients with mesenteric ischemia. A further multi-center prospective study with a larger sample size needs to be performed to confirm these findings.

\section{Acknowledgments}

Funding: None.

\section{Footnote}

Reporting Checklist: The authors have completed the STROBE reporting checklist. Available at http://dx.doi. org/10.21037/atm-20-8154

Data Sharing Statement: Available at http://dx.doi. org/10.21037/atm-20-8154

Conflicts of Interest: All authors have completed the ICMJE uniform disclosure form (available at http://dx.doi. org/10.21037/atm-20-8154). The authors have no conflicts of interest to declare.

Ethical Statement: The authors are accountable for all aspects of the work in ensuring that questions related to the accuracy or integrity of any part of the work are appropriately investigated and resolved. All procedures performed in this study involving human participants were in accordance with the Declaration of Helsinki (as revised in 2013). This study was approved by the Ethics Committee of Beijing Tsinghua Changgung Hospital (No. 19033-0-01). Informed consent was obtained from each patient prior to their involvement in the study.

Open Access Statement: This is an Open Access article distributed in accordance with the Creative Commons Attribution-NonCommercial-NoDerivs 4.0 International License (CC BY-NC-ND 4.0), which permits the noncommercial replication and distribution of the article with the strict proviso that no changes or edits are made and the original work is properly cited (including links to both the formal publication through the relevant DOI and the license). See: https://creativecommons.org/licenses/by-nc-nd/4.0/.

\section{References}

1. Clair DG, Beach JM. Mesenteric Ischemia. N Engl J Med 2016;374:959-68.

2. Karkkainen JM, Acosta S. Acute mesenteric ischemia (part I) - Incidence, etiologies, and how to improve early diagnosis. Best Pract Res Clin Gastroenterol 2017;31:15-25.

3. Groesdonk HV, Raffel M, Speer T, et al. Elevated endothelin-1 level is a risk factor for nonocclusive mesenteric ischemia. J Thorac Cardiovasc Surg 2015;149:1436-42 e2.

4. Bala M, Kashuk J, Moore EE, et al. Acute mesenteric ischemia: guidelines of the World Society of Emergency Surgery. World J Emerg Surg 2017;12:38.

5. Blumberg SN, Maldonado TS. Mesenteric venous thrombosis. J Vasc Surg Venous Lymphat Disord 2016;4:501-7.

6. Kumar S, Sarr MG, Kamath PS. Mesenteric venous thrombosis. N Engl J Med 2001;345:1683-8.

7. Harnik IG, Brandt LJ. Mesenteric venous thrombosis. Vasc Med 2010;15:407-18.

8. Zarrouk M, Salim S, Elf J, et al. Testing for thrombophilia in mesenteric venous thrombosis - Retrospective original study and systematic review. Best Pract Res Clin Gastroenterol 2017;31:39-48.

9. Russell CE, Wadhera RK, Piazza G. Mesenteric venous thrombosis. Circulation 2015;131:1599-603.

10. AbuRahma AF, Stone PA, Srivastava M, et al. Mesenteric/ celiac duplex ultrasound interpretation criteria revisited. J Vasc Surg 2012;55:428-36.e6; discussion 435-6.

11. Hagspiel KD, Flors L, Hanley M, et al. Computed tomography angiography and magnetic resonance angiography imaging of the mesenteric vasculature. Tech Vasc Interv Radiol 2015;18:2-13.

12. Iain D. C. Kirkpatrick MAK, Howard M. Greenberg. Biphasic CT with Mesenteric CT Angiography in the Evaluation of Acute Mesenteric Ischemia: Initial Experience. Radiology 2003;229:91-8.

13. Arthurs ZM, Titus J, Bannazadeh M, et al. A comparison of endovascular revascularization with traditional therapy for the treatment of acute mesenteric ischemia. J Vasc Surg 2011;53:698-704; discussion 704-5.

14. Bjornsson S, Resch T, Acosta S. Symptomatic mesenteric atherosclerotic disease-lessons learned from the diagnostic workup. J Gastrointest Surg 2013;17:973-80.

15. Oliva IB, Davarpanah AH, Rybicki FJ, et al. ACR Appropriateness Criteria (R) imaging of mesenteric ischemia. Abdom Imaging 2013;38:714-9. 
16. Duran R, Denys AL, Letovanec I, et al. Meuli, Sabine Schmidt. Multidetector CT Features of Mesenteric Vein Thrombosis. Radiographics 2012;32:1503-22.

17. Kanasaki S, Furukawa A, Fumoto K, et al. Acute Mesenteric Ischemia: Multidetector CT Findings and Endovascular Management. Radiographics 2018;38:945-61.

18. Glenister KM, Corke CF. Infarcted Intestine: A diagnostic Void. ANZ J Surg 2004;74:260-5.

19. Kim HK, Hwang D, Park S, et al. Treatment outcomes and risk factors for bowel infarction in patients with acute superior mesenteric venous thrombosis. J Vasc Surg Venous Lymphat Disord 2017;5:638-46.

20. Nuzzo A, Maggiori L, Ronot M, et al. Predictive Factors of Intestinal Necrosis in Acute Mesenteric Ischemia: Prospective Study from an Intestinal Stroke Center. Am J Gastroenterol 2017;112:597-605.

21. Wang X, Chu C, Sun S, et al. Outcomes and clinical characteristics of transmural intestinal necrosis in acute mesenteric ischemia. Scand J Gastroenterol 2019;54:953-9.

Cite this article as: Zhao $\mathrm{H}$, Meng $\mathrm{Y}$, Zhang $\mathrm{P}$, Zhang Q, Wang F, Li Y. Predictors and risk factors for intestinal necrosis in patients with mesenteric ischemia. Ann Transl Med 2021;9(4):337. doi: 10.21037/atm-20-8154
22. Corcos O, Castier Y, Sibert A, et al. Effects of a multimodal management strategy for acute mesenteric ischemia on survival and intestinal failure. Clin Gastroenterol Hepatol 2013;11:158-65.e2.

23. Wang Y, Zhao R, Xia L, et al. Predictive Risk Factors of Intestinal Necrosis in Patients with Mesenteric Venous Thrombosis: Retrospective Study from a Single Center. Can J Gastroenterol Hepatol 2019;2019:8906803.

24. Corcos O, Nuzzo A. Gastro-intestinal vascular emergencies. Best Pract Res Clin Gastroenterol 2013;27:709-25.

25. Wyers MC. Acute mesenteric ischemia: diagnostic approach and surgical treatment. Semin Vasc Surg 2010;23:9-20.

26. Brandt LJ BS. AGA Technical Review on Intestinal Ischemia. Gastroenterology 2000;118:954-68.

27. Singal AK, Kamath PS, Tefferi A. Mesenteric venous thrombosis. Mayo Clin Proc 2013;88:285-94. 